\title{
Sonographic Detection of Fetal Extrathoracic Pulmonary Sequestration
}

\author{
F. Mariona, $M D_{i}{ }^{*}$ G. McAlpin, MD, $\neq$ I. Zador, PhD, ${ }^{*} A$. Philippart, MD, $\neq$ \\ S. Z. H. Jafri, MD†
}

The use of diagnostic ultrasonography during pregnancy has gained widespread acceptance. Unquestionably this diagnostic modality facilitates discovery of fetal anomalies that might otherwise remain unsuspected. Such discoveries can produce therapeutic dilemmas, but may permit the obstetrician to tailor antepartum and intrapartum care in accordance with potential deleterious effects of the anomaly on the fetus or newborn, and the emotional disturbance that such discovery provokes in patients and family.

We report a case of retroperitoneal pulmonary sequestration that was diagnosed during the antenatal period.

\section{REPORT OF A CASE}

A 23-year-old black woman, gravida 4, para 2, abortus 1 , lad two previous uneventfil pregnancies with normal full-term spontaneous vaginal deliveries. At 37 weeks" gestation by dates she was referred for ultrasound exatmination because of questionable intppropriate size for dates. The examination showed a single, living, intrauterine fetus of 34 weeks' size. Fetal head to body ratio was normal, and femur length concurred with fetal age. A large retroperitoneal mass measuring approximately $3.5 \mathrm{~cm}$ in diameter was noted in the right upper quadrant. It was uniformly echogenic with smooth margins (fig. 1). Fetal kidneys, stomach, liver, and diaphragm were visualized as normal. The fetus was in vertex presentation with a grade II posterior placenta. Amniotic fluid volume was normal, and no ascites was noted. Follow-up examination three days later showed no change in the size and appearance of the mass. Because of the location of the tumor and the clate in pregnancy at which the discovery was made, the diagnosis of a neuroblastoma was entertained. An atterm vagioal delivery in a tertiary care level institution was planned, and a pediatric surgeon was consulted. The parents agreed to a conservative treatment approach since no obvious deterioration of fetal health was evident. The patient's membranes ruptured prematurely one week after the ultrasound examination, and she spontaneously deliv-

Received September 28, 1984, from the "Departments of Obstettics/Gynecology and TRadiology, Hutzel Hosptal, Wayne State University School of Medicine. Detroit. Michigan, and the $\neq$ Department of Surgery, Children's Hospital of Mich gan. Wayne State University School of Medicine, Detroit, Michigan. Revised manuscript accepted for publication October 25, 1985

Address correspondence and reprint requests to Dr. Mariona: Division of Maternal-Fetal Medicine, Department of OB/GYN. Hutzel Hospital, 4707 St. Antoine, Detroit. MI 48201. ered an infant weighing 2466 grams, appropriate for gestational age, witl Apgar scores of 8 and 9 at one and five minutes, respectively. The Ballard score corresponded to 34 weeks.

Plyysical examination of the newborn revealed no palpable abdominal mass. Chest, abdominal, and longbone radiographs were normal. Intravenous pyelography showed flattening and slight inferior displacement of the right kidney consistent with a suprarenal mass. Ultrasound examination revealed a well-circumscribed, rather homogenously echogenic mass in the right suprarenal position. The mass appeared separate from the kidney, liver, and inferior venia cava with compression of the latter. These findings were confirmed by computed tomography (Figs. $2 A$ and $B$ ).

The infunt underwent exploratory surgery through a right subcostal incision. The mass was inferior and adherent to the liver, arising from the superior surface of an otherwise normal appearing adrenal gland. It was soft, pinkish in color, and solid. The arterial supply was systemic mainly from the right renal artery through the adrenal gland, and one large and several smaller branches from the aorta to the mass. Additional appreciable blood supply was derived from the inferior phrenic artery. Venous return was to the inferior vena cava through "normal" adrenal veins. No communication to the gastrointestinal tract was present. Because of the adherence of the mass to the adrenal gland and the common blood supply, an en bloc resection including the right adrenal was performed.

Pathologic examination revealed an ovoid, spongy, pink-gray mass, measuring $4 \mathrm{~cm}$ in dianeter, weighing $12 \mathrm{~g}$, and consisting of lung parenchyma at varying stages of maturity. Bronchioles lined with ciliated respiratory epithelium were seen. These findings were consistent with extralobar sequestered lung in the periadrenal location.

\section{DISCUSSION}

Pulmonary sequestration is a rare congenital anomaly of the developing lung which was first described over 100 years ago. The term was introduced by Pryce who described an ectopic, nonfunctioning pulmonary mass which lacked communication with the tracheobronchial tree and received blood supply from an anomalous systemic vessel. " The two classical forms of sequestration are: 1) intralobar, denoting a lesion that lies within the pulmonary visceral pleura, and 2) extralobar, denoting a lesion which lies outside the pulmonary visceral pleura. The arterial supply to an intralobar sequestration is systemic with pulmonary venous drainage, whereas extralobar lesions usually have 


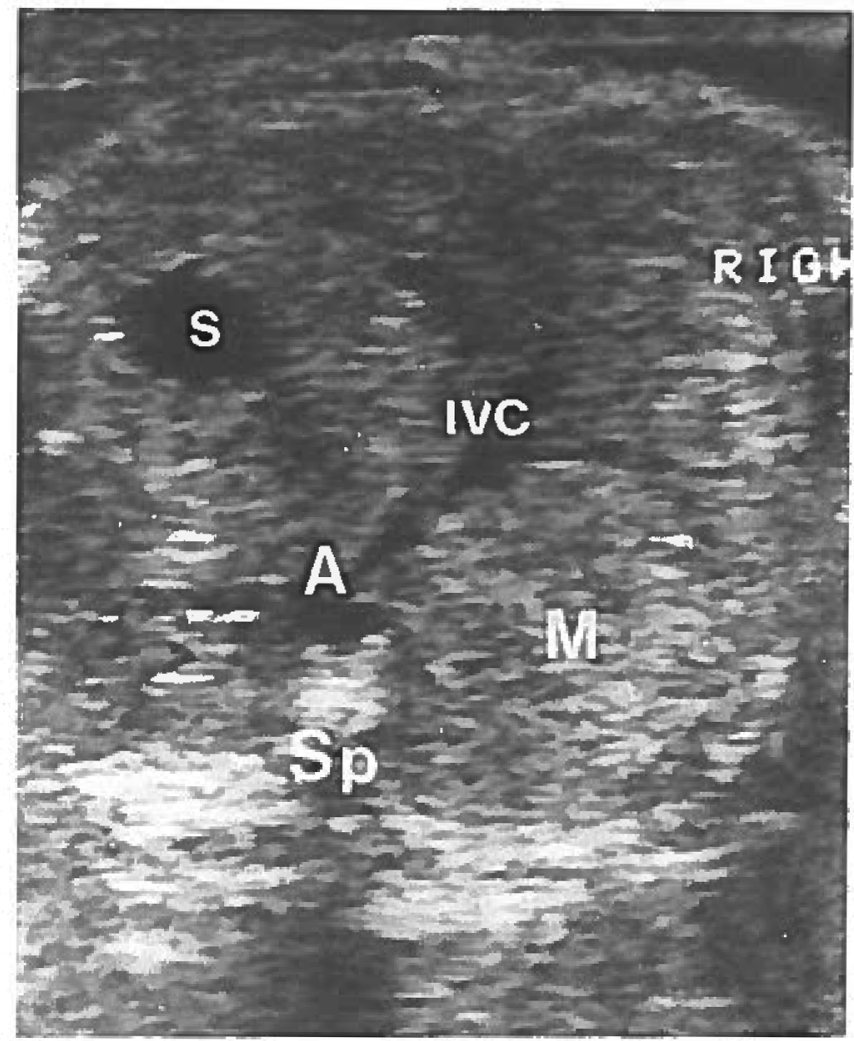

Figure 1 (left). Transverse section through fetal abdomen at the level of the fetal stomach showing the retroperitoneal mass. $M$, mass; SP, spine; $A_{t}$ aorta; IVC, inlerior vena cava; s, stomach.

Figure 2 A (lower left). Unenhanced computed tomographic (CT) scan of the neonatal abdomen demonstrates a low attenuating mass in the right upper quadrant. No calcifications are seen within the mass. $M_{3}$ mass. $B$ (lower right), enhanced CT scan of the neonatal abdomen demonstrates a mass with peripheral and focal enhancement displacing the inferior vena cava anteriorly. $M$, mass; $A o$, aorta: IVC, inferior vena cava.

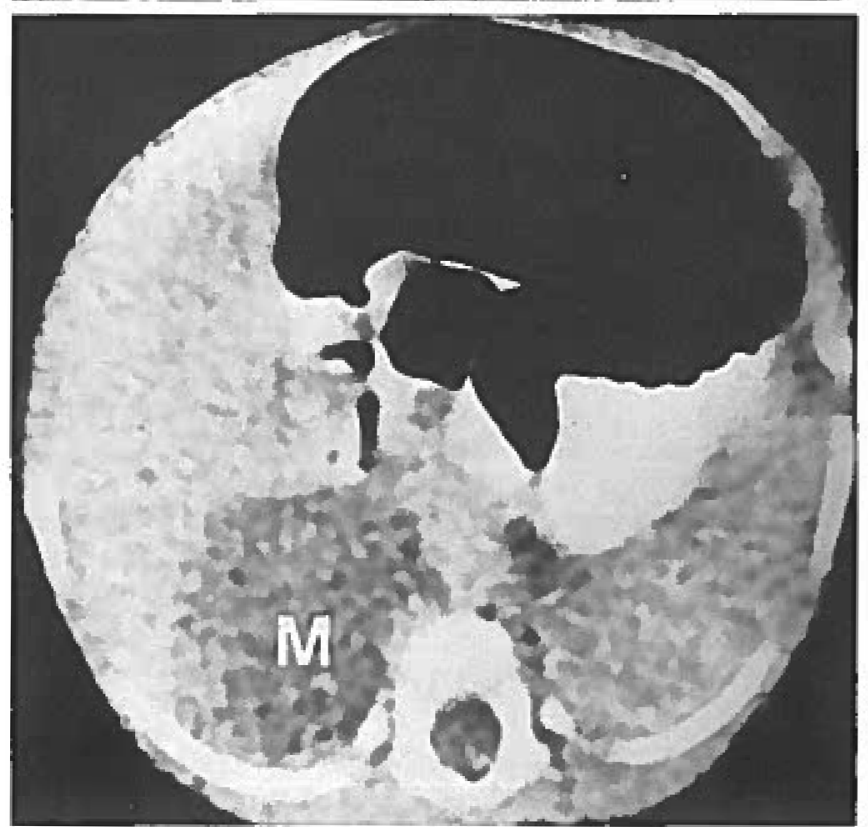

both systemic arterial and venous supply. Although the etiology of pulmonary sequestration remains controversial, it is accepted that extralobar and intralobar sequestrations are bronchopulmonary foregut malformations. ${ }^{2}$ Tiventy-five per cent of pulmonary sequestrations are extralobar, ${ }^{3,4}$ the miljority of these are left-sided with male predominance. Approximately $\overline{5}$ per cent are located below the diaphringm. 5,6

The present case depicts an extralobat pulmo-

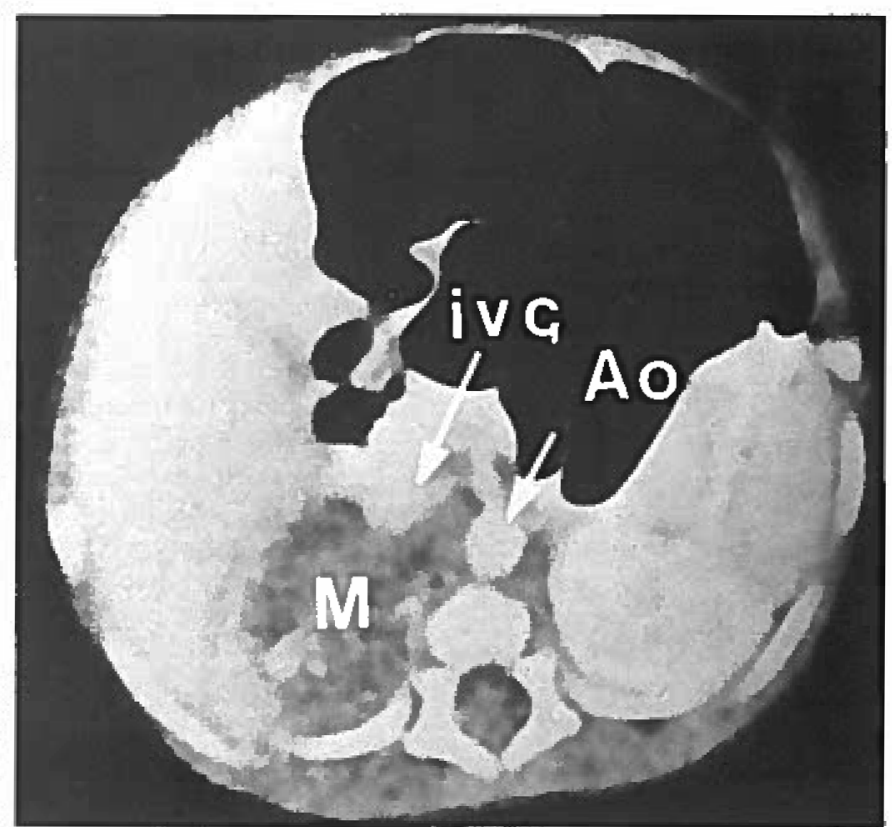

nary sequestration located in the right suprarenal position. It was first identified prenatally by ultrasonography, thus enabling serial examinations of the lesion and the taking of adequate precautions at delivery. It appeared to be a solid, dense mass with a few hypoechogenic areas. It displaced the right kidney inferiorly and inferior vena cava anteriorly and towards the midline (figs. $I$ and $2 B$ ). The incorrect initial working diagnosis was a result, in part, of the location of the mass. The most common le- 
sion in the differential diagnosis given this presentation would be neuroblastoma. Adrenal hemorrhage has been thought to be a postnatal event and to our knowledge not previously recognized in utero. Retroperitoneal teratomats could occur in this area but would be anticipated to be more cystic in alppearance.

Pulmonary sequestration and diaphragmatic hernia must be considered with the need for anggressive neoniatal support for the latter. Knowledge of the sonographic appearance for postnital iclentification of a pulmonary sequestration by ultrasisnography is essential since the clinical and radiologic appearance may mimic other lesions such as cystic adenomatoid malformation or bronchogenic cyst. In conclusion, accurate antentatal diagnosis of pulmonary secjuestration is feasible. This permits adoption of precautionary measures to deal with any postnatal complications including congestive heart failure, or, rarely, respiratory distress when shunting is present, 7.8 Conservative therapy in the event of pulmonary sequestration can be considered if there is no evidence of fetal cleterioration.

\section{REFERENCES}

1. Pryce $D M$, et al: Intralobular sequestration of lung associated with an abnormal pulmonary artery $\mathrm{Br} \mathrm{J}$ Surg 3518,1947

2. Gerle RD, et al: Congental bronchopulmonary-foregut malformation. N Engl J Med 278:1413, 1968

3. DeParedes $C G$ el al: Pulmonary sequestration in infants and children a 20 year experience and review of the literature. $J$ Pediatr Surg 5-136, 1970

4. Butel SFJ, et al: Lung sequestration report of seven cases and review of 540 published cases. Thorax $34: 96,1979$

5. Buntain WL. et al: Pulmonary sequestration in children: a twenty-five year experience Surgery 81:413, 1977

6. Carter R. Pulmonary sequestration. Ann Thorac Surg 7:68. 1969

7. Werthammer JW. et al: Upper thoracic extralobar pulmonary sequestration presenting with respiratory distress in a newborn. Pediatr Radiol 9:116, 1980

8. Levine $M M$, et at Pulmonary sequestration causing congestive heart failure in infancy a report of two cases and review of the literalure Ann Thorac Siurg 34 581, 1982 